Shall we do this together? Social gaze influences action control in a comparison group, but not in individuals with high-functioning autism

Leonhard Schilbach, Simon B. Eickhoff, Edna C. Cieslik, Bojana Kuzmanovic and Kai Vogeley Autism 2012 16: 151 originally published online 2 August 2011

DOI: $10.1177 / 1362361311409258$

The online version of this article can be found at:

http://aut.sagepub.com/content/16/2/151

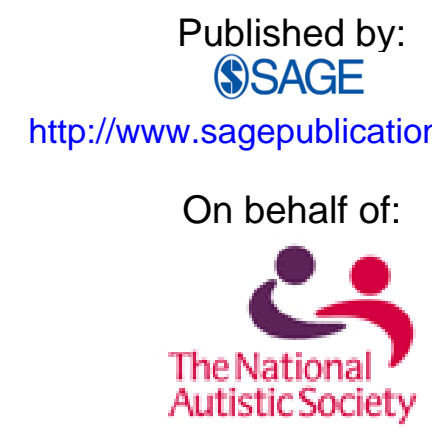

The National Autistic Society

Additional services and information for Autism can be found at:

Email Alerts: http://aut.sagepub.com/cgi/alerts

Subscriptions: http://aut.sagepub.com/subscriptions

Reprints: http://www.sagepub.com/journalsReprints.nav

Permissions: http://www.sagepub.com/journalsPermissions.nav

Citations: http://aut.sagepub.com/content/16/2/151.refs.html

>> Version of Record - Mar 15, 2012

OnlineFirst Version of Record - Aug 2, 2011

What is This? 
Shall we do this together? Social gaze influences action control in a comparison group, but not in individuals with high-functioning autism
16(2) $15 \mid-162$

(C) The Author(s) 2012

Reprints and permission: sagepub.co.uk/journalsPermissions.nav DOI: $|0.1| 77 /|36236| 3|| 409258$ aut.sagepub.com

@SAGE

\title{
Leonhard Schilbach
}

Max-Planck Institute for Neurological Research, Cologne, and University of Cologne, Germany

\section{Simon B. Eickhoff}

Research Centre Juelich, and University of Aachen, Germany

\section{Edna C. Cieslik}

Research Centre Juelich, Germany

\section{Bojana Kuzmanovic}

University of Cologne, Germany

\section{Kai Vogeley}

University of Cologne, and Research Centre Juelich, Germany

\begin{abstract}
Perceiving someone else's gaze shift toward an object can influence how this object will be manipulated by the observer, suggesting a modulatory effect of a gaze-based social context on action control. High-functioning autism (HFA) is characterized by impairments of social interaction, which may be associated with an inability to automatically integrate socially relevant nonverbal cues when generating actions. To explore these hypotheses, we made use of a stimulusresponse compatibility paradigm in which a comparison group and patients with HFA were asked to generate spatially congruent or incongruent motor responses to changes in a face, a face-like and an object stimulus. Results demonstrate that while in the comparison group being looked at by a virtual other leads to a reduction of reaction time costs associated with generating a spatially incongruent response, this effect is not present in the HFA group. We suggest that this
\end{abstract}

Corresponding author:

Leonhard Schilbach, Max-Planck-Institute for Neurological Research, Gleueler Str. 50, 5093I Cologne, Germany.

Email: leonhard.schilbach@nf.mpg.de 
modulatory effect of social gaze on action control might play an important role in direct social interactions by helping to coordinate one's actions with those of someone else. Future research should focus on these implicit mechanisms of interpersonal alignment ('online' social cognition), which might be at the very heart of the difficulties individuals with autism experience in everyday social encounters.

\section{Keywords}

action control, high-functioning autism, social gaze, stimulus-response compatibility

When someone observes another individual shifting gaze, the observer's attention automatically and rapidly orients to the same location (Emery, 2000). This can affect the perception of an object located in the line of gaze (Becchio et al., 2008; Bayliss et al., 2006) or of the person having produced the gaze shift (Macrae, 2002; Kuzmanovic et al., 2009). Furthermore, gaze cues have been shown to trigger mechanisms of motor facilitation by which interactors assimilate their motor behavior and generate preparatory motor responses that can be helpful in predicting someone else's behavior (Cattaneo et al., 2007). Similarly, it has also been demonstrated that gaze shifts towards objects can evoke processes, which in turn modulate the manipulation of objects (Becchio et al., 2007).

Autism is characterized by impairments of social interaction and communication which are assumed to be related to difficulties in responding to socially relevant nonverbal cues. In this respect, it has recently been stressed that in high-functioning autism (HFA) important differences may exist between an inability to grasp and automatically integrate gaze cues and intact capabilities of making a conscious and explicit effort to evaluate social information (Senju et al., 2009). This appears to be in line with the idea that social cognition may be fundamental when we are actively engaged in interaction with others ('online' social cognition) as compared to merely observing others ('offline' social cognition; Schilbach, 2010). This difference may be particularly relevant for HFA and could be taken to suggest that 'online' social cognition relies more heavily on the integration of perception- and action-based processes. While there is, in fact, ample evidence for atypical gaze patterns and alterations of gaze perception in autism (Klin et al., 2003), much less is known about the putative interaction of mechanisms of gaze processing and motor control in autism, even though impairments of both are known to be characteristic features of autism (Rinehart et al., 2006).

To explore the possible modulatory effect of social gaze on mechanisms of action control, we made use of a novel experimental paradigm (Schilbach et al., 2010), which allows for the investigation of the effect of a social context provided by gaze cues on the generation of rapid manual responses in adults with HFA and a matched group of participants. In this stimulus-response compatibility (SRC) task participants are asked to generate motor responses (button presses), which are spatially congruent or incongruent (response type: CON vs. INC) to changes in a visual stimulus occurring either towards the left or right. As visual stimuli, the face of an anthropomorphic virtual character, a face-like stimulus and a non-social stimulus were used (stimulus type: SOC vs. INT vs. OBJ; see Figure 1), thereby constituting a $2 \times 3$ factorial design.

Traditionally, non-social SRC tasks have provided evidence for incongruency costs both with respect to reaction times (RT) and to the percentage of correct responses (CR), demonstrating an increase of RT and decrease of CR for incongruent as compared to congruent motor responses (e.g. Proctor and Reeve 1990; Iacoboni et al., 1996; Matsumoto et al., 2004). In our 'social' SCR task, we expected to observe a modulatory effect of the stimulus type on the resulting incongruency 
A

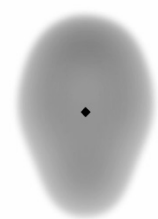

A

B

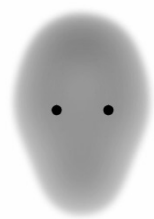

C
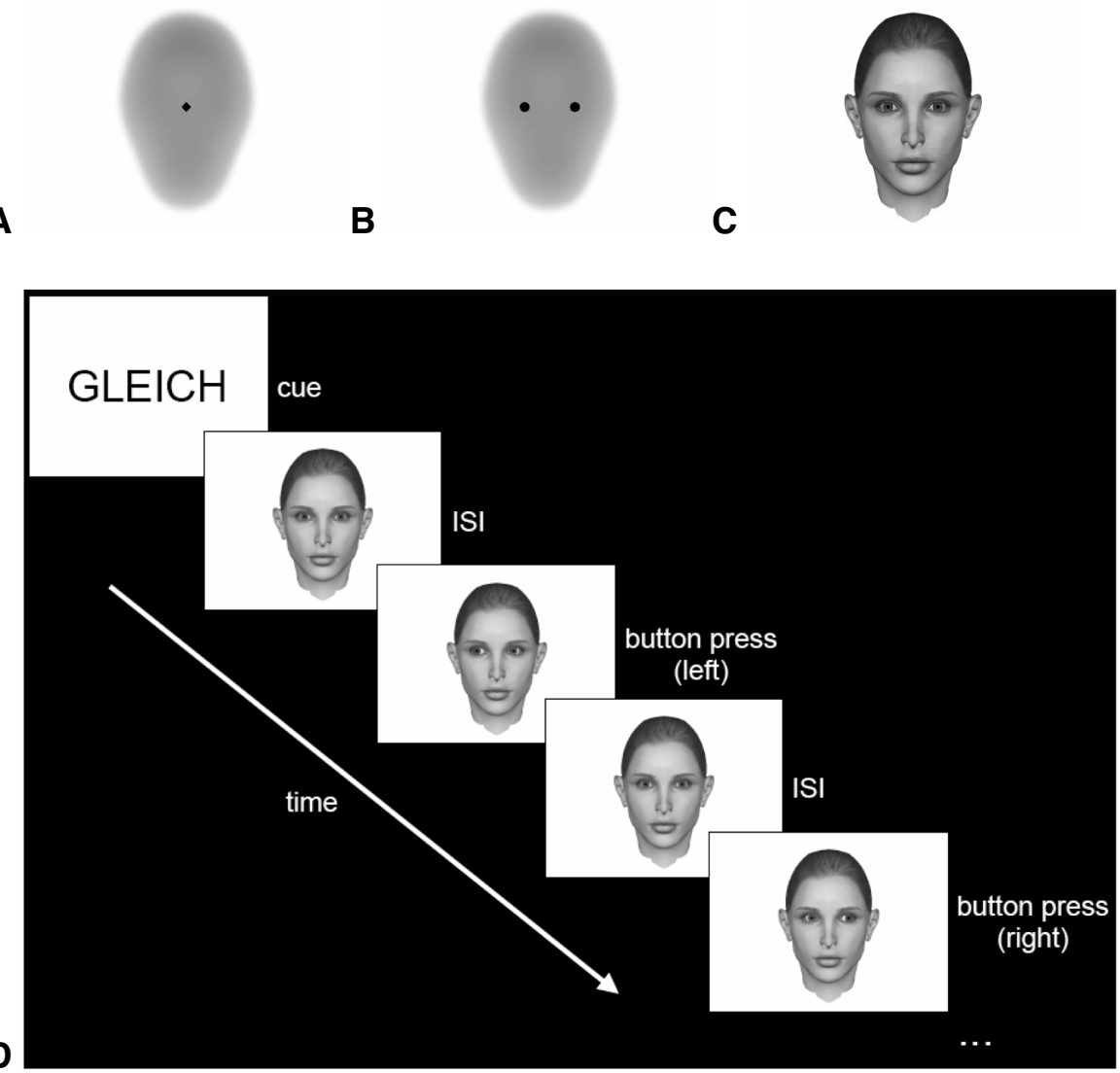

Figure I. Social (A;'OBJ') and non-social stimuli (B, C;'INT', 'SOC') used in the study. (D) Exemplary depiction of the event structure for congruent responses to the SOC stimulus. ISI: interstimulus interval.

costs: in the control group, we hypothesized that incongruent responses to a social stimulus would lead to more pronounced incongruency costs than responses to the non-social stimuli, based on previous research suggesting that healthy controls have a tendency to reflexively follow gaze shifts which may increase reaction times for contralateral motor responses (Emery, 2000; Ristic and Kingstone, 2005; Shepherd, 2010). Conversely, and in light of evidence suggestive of an 'immunity' to the modulatory effects of gaze stimuli on action control in autism (Becchio et al., 2007), we expected that the incongruency effect within the HFA group would be less affected by the stimulus type.

\section{Method}

\section{Participants}

Fifty-seven participants participated in this study, which was conducted with the approval of the local ethics committee of the Medical Faculty at the University of Cologne. Twenty-nine patients with HFA (mean age 32.0, SD = 9.2 years, 17 male, 12 female) were age and intelligence (IQ) matched with twenty-eight healthy participants (mean age 29.96, SD $=4.66$ years, 12 male, 16 female; see Table 1). IQ was assessed by a German multiple choice vocabulary test ('Wortschatztest', 
Table I. Demographic and neuropsychological variables of control and patient group

\begin{tabular}{|c|c|c|c|c|c|c|}
\hline & \multicolumn{2}{|c|}{ HFA group } & \multicolumn{2}{|c|}{ Control group } & \multirow[t]{2}{*}{ Statistics } & \\
\hline & $M$ & SD & $M$ & SD & & \\
\hline Age (in years) & 32.00 & 9.22 & 29.96 & 4.66 & $t(55)=-1.046$ & $p=.296$ \\
\hline IQ & 110.70 & 13.07 & 113.93 & 11.34 & $t(55)=.998$ & $p=.322$ \\
\hline $\mathrm{BDI}^{\mathrm{a}}$ & 17.93 & 12.07 & 1.25 & 1.32 & $t(55)=-7.4$ & $p<.001$ \\
\hline $\mathrm{AQ}^{\mathrm{a}}$ & 38.17 & 5.52 & 14.07 & 4.74 & $t(55)=-17.643$ & $p<.001$ \\
\hline
\end{tabular}

Note. HFA = high-functioning autism; $M=$ Mean; SD = standard deviation; BDI = Beck Depression Inventory; $\mathrm{AQ}=$ Autism Quotient.

a. Raw score (not standardized).

WST) (Schmidt and Metzler, 1992), which allows for a quick and valid estimation of general intelligence (Lehrl et al., 1995; Suslow, 2009). The group of participants for comparison with the patients reported no history of neurological or psychiatric disorders, and no current use of any psychoactive medications. Furthermore, they were only included if they had an Autism Spectrum Quotient (AQ) below 23 (Baron-Cohen, 2003) and a Beck Depression Inventory (BDI) score of 17 or below (Beck and Steer, 1987). All patients with HFA were diagnosed and recruited in the Autism Outpatient Clinic at the Department of Psychiatry at the University of Cologne (Germany). As part of a systematic assessment implemented in this clinic, diagnoses were made by specialized clinicians corresponding to ICD-10 criteria and supplemented by an extensive neuropsychological assessment. Patients with the diagnosis of Asperger's syndrome (F84.5) and childhood autism (F84.0) were included when average or above-average IQ had been ascertained. All patients, therefore, belonged to the high-functioning autism population. Due to the fact that both diagnostic groups exhibit a comparable intellectual level of functioning and that differences in diagnostic criteria relate to language development in early childhood irrelevant for the adult level of social adaptation, the two diagnoses were subsumed under the term HFA. As depression is a common co-morbidity in HFA (e.g. Stewart et al., 2006) and due to mood disturbance serving as an exclusion criterion in the comparison group, it does not come as a surprise that there was a significant difference in the BDI score for the control and the HFA group (see Table 1). Consistent with the clinical diagnoses, there were also significant differences in the AQ between the patient and the comparison group (see Table 1) (Wheelwright et al., 2006).

\section{Experimental protocol}

Before participation, all participants received standardized instructions and were familiarized with the task. Participants were instructed to respond as quickly and correctly as possible to each change of the target stimulus by pressing a button according to the task condition. The change in target stimulus could either be (a) a gaze shift towards the right or left shown by an anthropomorphic virtual character which otherwise looked straight ahead (SOC; see Figure 1A and D); (b) the displacement of two dots of the same size as the pupils of the face towards the left or right (INT; see Figure 1B); or (c) the displacement of a square of the same size as the pupil of the face towards the left or right from a central starting position (OBJ; see Figure 1C). Using a paradigm which explicitly requires participants to attend to the gaze direction of the face is helpful for use in autism, because it minimizes the possibility of reduced fixations of the eye region in patients. The offset in 
pixel coordinates and the timing of the movements within the stimuli were equivalent in all conditions. To keep visual input similar across conditions, the dot and square stimuli were shown in front of a smoothed face as a background. For the congruent condition (CON) participants were instructed to respond with the ipsilateral hand (i.e. pressing a button with their left index finger to a left-moving stimulus and with their right index finger to a right-moving stimulus). For the incongruent condition (INC) participants were instructed to respond with the contralateral hand (i.e. pressing another button with their left index finger to a right-moving stimulus and with their right index finger to a left-moving stimulus). Both response buttons were made available on a computer keyboard. Visual stimuli were presented using the software package Presentation (Version 11.3) on a laptop computer at $1024 \times 768$ pixels and a frame rate of $100 \mathrm{~Hz}$. Viewed from a distance of $80 \mathrm{~cm}$ while seated in front of the computer, the screen subtended a visual angle of $28^{\circ}$ horizontally and $19^{\circ}$ vertically.

During the experiment, task blocks lasting $57-63 \mathrm{~s}$ were periodically alternated with rest periods which lasted 4.5 to $7.5 \mathrm{~s}$. Each task block started with an instruction cue, either the German word 'GLEICH' ('SAME') for the congruent condition or the German word 'GEGEN' ('OPPOSITE') for the incongruent condition, being presented for $1500 \mathrm{~ms}$ to inform the subject which of the two experimental conditions had to be performed throughout the subsequent block (see Figure 1D). Regardless of the condition, 12 events per block (50\% left- and 50\% right-moving stimuli presented at random) occurred. The interstimulus interval (ISI) was uniformly jittered between 2.5 and $7 \mathrm{~s}$. In the course of the entire experiment, all experimental conditions were presented in 24 blocks whose order was pseudo-randomized and counterbalanced across subjects.

\section{Data analysis}

The dependent variables were the reaction time (RT) of the responses given and the percentage of correct responses (CR). The behavioral measurements obtained during the experiment were preprocessed using MATLAB (Math-Works, Natick, MA) and analysed by using the Predictive Analytic Software 18 (PASW18; www.spss.com). Responses with latencies of less than $150 \mathrm{~ms}$ or greater than $1600 \mathrm{~ms}$ were regarded as anticipation errors or missed responses, respectively, and discarded from the later analysis. We calculated the incongruency costs for each participant by subtracting RTs and CRs for the congruent responses to each stimulus type from the corresponding measurement for the incongruent response, respectively. Here, we noted that both control and patient group demonstrate a statistically significant effect of incongruency with spatially incongruent responses leading to longer RTs and lower CRs as assessed by means of a repeated measures analysis of variance (rmANOVA; see Results for details). The difference values of RT ('RT costs') and CR ('CR costs') for each participant were used in further statistical analyses in which the effect of the within-subject independent variable stimulus type was compared by means of a mixed rmANOVA with a between-subject independent variable group (HFA vs. control) and dependent variables RT costs or CR costs, respectively.

Due to the fact that the comparison and patient group consisted of different numbers of female and male participants, we also performed a mixed rmANOVA which employed both the betweensubject independent variable group (HFA vs. control) and the between-subject independent variable gender (male vs. female) to investigate possible gender-specific differences in task performance. In order to rule out a possible influence of BDI scores on task performance, the BDI scores were used for correlation analyses with RT and CR measurements across all experimental conditions. 
Table 2. Descriptive statistics of reaction times, percentages of correct responses and incongruency costs across all experimental conditions and groups

\begin{tabular}{lcccccc}
\hline \multicolumn{7}{c}{ Experimental conditions } \\
\cline { 2 - 7 } & SOC_CON & SOC_INC & INT_CON & INT_INC & OBJ_CON & OBJ_INC \\
\hline Control group & & & & & & \\
RT [ms] & $496(83)$ & $55 I(87)$ & $529(95)$ & $589(85)$ & $508(87)$ & $593(101)$ \\
CR [\%] & $96(5)$ & $92(7)$ & $92(6)$ & $81(15)$ & $92(14)$ & $86(13)$ \\
RT cost [ms] & $56(32)$ & $60(55)$ & $85(6 I)$ & & & \\
CR cost [\%] & $3(1)$ & $10(2)$ & $6(1)$ & & & \\
HFA group & & & & & \\
RT [ms] & $532(113)$ & $618(134)$ & $55 I(114)$ & $626(122)$ & $545(104)$ & $614(122)$ \\
CR [\%] & $86(2 I)$ & $8 I(23)$ & $87(13)$ & $78(19)$ & $88(12)$ & $80(17)$ \\
RT cost [ms] & $86(78)$ & $74(63)$ & $70(63)$ & & & \\
CR cost [\%] & $5(1)$ & $9(2)$ & $8(2)$ & & & \\
\hline
\end{tabular}

Note. Means and standard deviations are shown in brackets. $\mathrm{RT}=$ reaction times; $\mathrm{CR}=$ correct responses; cost $=$ incongruency costs representing the difference between congruent and incongruent condition; HFA = high-functioning autism; SOC = face stimulus; INT = face-like stimulus; OBJ = object stimulus; $C O N=$ congruent; INC = incongruent.

\section{Results}

Mean RTs, mean percentages of CR and mean RT/CR costs of the control and HFA group are summarized in Table 2. We observed incongruency effects for RTs and CRs in both control (RT: F(1, $27)=76.61, p<.001 ; \mathrm{CR}: \mathrm{F}(1,27)=19.45, p<.001)$ and HFA group $(\mathrm{RT}: \mathrm{F}(1,28)=57.75$, $p<.001 ; \mathrm{CR}: \mathrm{F}(1,28)=10.37, p=.003)$, indicating significantly longer RTs and lower percentages of CRs for incongruent responses.

Mixed rmANOVAs employing the within-subject factor stimulus type (SOC vs. INT vs. OBJ) and a between-subject independent variable group (HFA vs. control) were performed to assess group-specific effects on RT and CR costs, respectively. For RT costs this analysis did neither demonstrate a significant effect of group $(\mathrm{F}(1,55)=2.00, p=.16)$ nor stimulus type $(\mathrm{F}(2,110)=$ $0.74, p=.48)$, but demonstrated a stimulus type $\times$ group interaction $(\mathrm{F}(2,110)=3.63, p=.03)$. To break down this interaction, simple effects were performed which demonstrated a significant effect of stimulus type on the RT costs in the control group $(\mathrm{F}(2,110)=3.34, p=.04)$, while no such effect was observed in the patient group $(\mathrm{F}(2,110)=0.99, p=.38)$. Here, it is noteworthy that in the control group the effect of stimulus type on RT costs was carried by the statistical difference between OBJ and SOC stimuli as demonstrated by pair-wise comparisons $(\mathrm{T}(27)=2.82, p=.01$, $\mathrm{r}=.31)$, while no significant difference between INT and SOC $(\mathrm{T}(27)=0.53, p=.59, \mathrm{r}=.14)$ and INT and OBJ $(\mathrm{T}(27)=2.16, p=.04, \mathrm{r}=.27)$ was observed when correcting for multiple testing (see Figure 2). In the patient group no significant differences were observed.

For CR costs the rmANOVA analysis neither demonstrated an effect of group $(\mathrm{F}(1,55)=0.09$, $p=.76)$, stimulus type $(\mathrm{F}(2,110)=2.40, p=.10)$ nor an interaction effect of stimulus $\infty$ group $(\mathrm{F}(2$, $110)=0.30, p=.71)$.

RmANOVA employing both the between-subject independent variable group (HFA vs. control participants) and the between-subject independent variable gender (male vs. female participants) replicated the above described findings for group-specific differences in task performance, but did not show any evidence of gender-specific differences, neither demonstrating a main effect of gender, nor any gender-specific statistical interaction. Correlation analyses of BDI scores with RT and CR across all experimental conditions did not show any significant results (maximum $\mathrm{r}=.188$, minimum $p=.162$ ). 


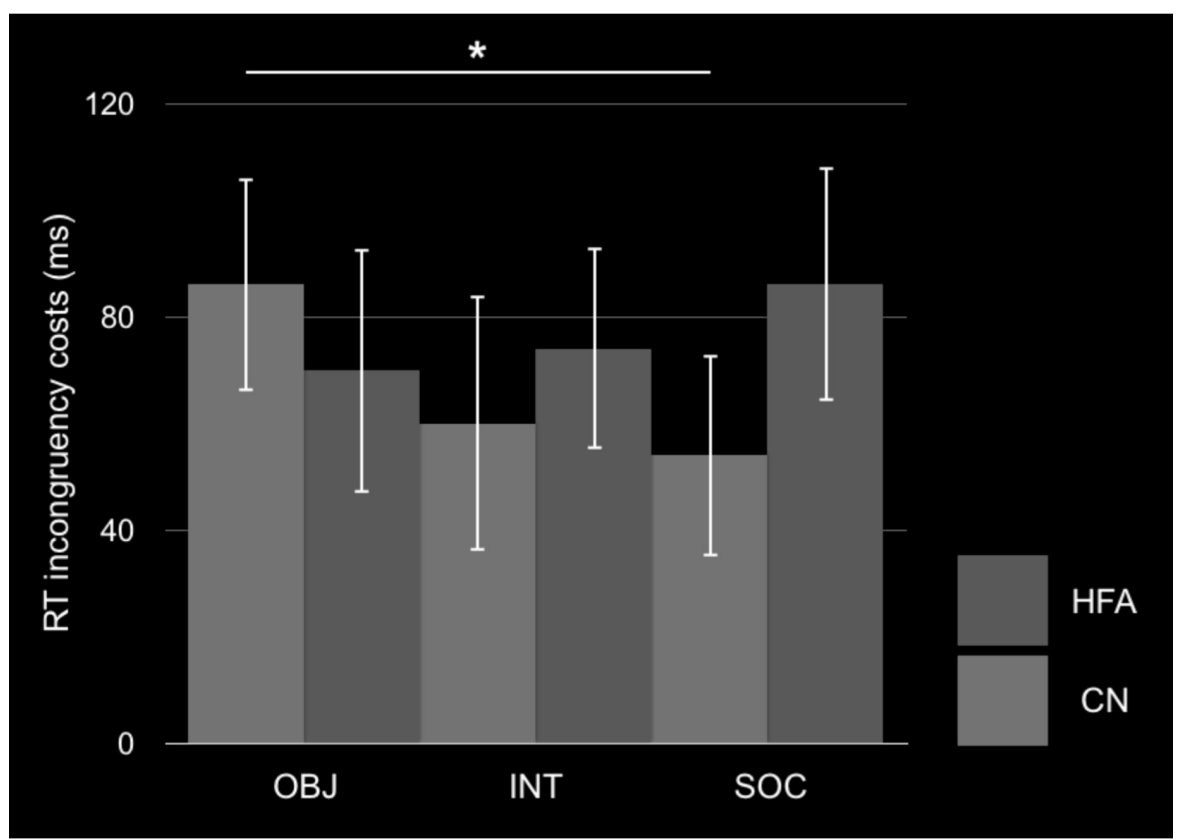

Figure 2. Bar graphs showing RT incongruency costs (RT difference between incongruent and congruent condition in $\mathrm{ms}$ ) for both control (A) and patient (B) group across all stimulus types (OB): object stimulus, INT: face-like stimulus, SOC: face stimulus). Error bars show $95 \%$ confidence intervals. Statistically significant difference as assessed by paired-sample $t$ test analysis highlighted by asterisk.

\section{Discussion}

We used a novel version of an SRC task to investigate the effect of a gaze-mediated social context on action control both in an adult comparison group and HFA participants based on the hypothesis that an influence of social context on motor performance can be observed in the comparison group, while such an effect is suspended in HFA in spite of intact explicit social cognitive abilities. Our data, indeed, corroborate this hypothesis by demonstrating a significantly reduced incongruency effect on RTs for the face stimulus in the comparison group, but indicate the absence of such an effect in the patient group.

\section{The modulatory influence of a gaze-based social context on action control}

Making use of a socially enriched SRC paradigm, we were able to replicate results from previous studies, which demonstrate an incongruency effect on RT and CR in control participants with incongruent responses leading to an increase of RT and a decrease of the number of CR (Proctor and Reeve, 1990; Iacoboni et al., 1996; Matsumoto et al., 2004). The RT differences between incongruent and congruent responses are deemed to reflect the extra 'computational load' associated with the inhibition of the reflexive congruent response, processes of attentional reorienting and the generation of the incongruent motor response. Importantly, however, we also observed that the stimulus type had a statistically significant impact on the performance of incongruent actions in the control group. Contrary to our initial hypothesis suggesting a more difficult and timeconsuming disengagement from a gaze cue - as a result of gaze cues being an evolutionarily 
significant and, therefore, potentially more powerful stimulus (von Grunau and Anston, 1995; Farroni et al., 2002; Grossmann et al., 2007; Hoehl et al., 2009; Senju and Johnson, 2009) - the results obtained indicate less-pronounced RT costs for the face as compared to the OBJ stimulus. In spite of the difference between OBJ and INT stimulus just failing to reach statistical significance, it seems noteworthy that in the comparison group this stimulus also leads to a substantial reduction of RT costs (Figure 2), which can be seen to highlight participants' sensitivity, even for face-like stimuli.

While our results, therefore, do not provide evidence for the hypothesis of more pronounced RT costs in response to gaze shifts in the comparison group, they still are consistent with the idea of a modulatory effect of a gaze-mediated social context on action control. In fact, it appears that participants' performance of incongruent motor actions actually benefits from the presence of a face stimulus. The latter could be seen to resonate with the idea of social facilitation (Zajonc, 1965), which suggests that the presence of others increases physiological arousal, which can have a performance-enhancing effect when the task is easy or familiar. Similarly, one might argue that a social stimulus such as a face might be perceived as more behaviorally relevant and could thereby lead to a general enhancement in the efficiency of processing operations (Quadflieg et al., 2004) and possibly to more pronounced action monitoring and inhibitory processes which could allow performance of incongruent responses more quickly than in the case of non-social stimuli. However, neither effect explains the differential modulation of task performance in response to social stimuli as observed in our study.

Consequently, one would have to assume an underlying mechanism, which explains how the presence of a virtual other specifically affects performance of a spatially incongruent action. Here, recent research investigating the effects of agency perception on what may be termed 'automatic perspective-taking' may be informative: in a study by Tversky and Hard (2009), participants were asked to describe spatial relations between objects in photographed scenes. In some versions, a person sitting behind the object was either looking at or reaching for one of the objects. Interestingly, the mere presence of another person in a position to act on the objects elicited descriptions of the spatial relations from that person's point of view from participants. Furthermore, when the question about the spatial relations was phrased in terms of action, more participants took the other person's perspective than their own. Similarly, in a study by Zwickel and colleagues (2009) it was shown that the processing of an agent's behavior - even if that agent is only a biologically moving triangle - automatically co-occurs with 'perspective adoption' and that the latter can be helpful to decide on which side of the agent an event occurs.

What these studies demonstrate is that the implication of action or behavioral cues suggestive of agency spontaneously elicits processes that have an impact on action control in a human observer. With respect to the paradigm used in our study, it makes sense to assume that similar effects were also elicited by the perception of an anthropomorphic virtual character directly looking at the participant before performing gaze shifts. We suggest that these effects may involve adjusting to the 'perspective' of the virtual other in terms of social affordances - that is, responding to the possibilities for action provided by a (gaze-mediated) social context (Costall, 1995). Consistent with this idea, it is conceivable that an action that is deemed spatially incongruent from an allocentric observer's point of view may end up being complementary to what the participant perceives the (virtual) other is doing. In other words: being in the presence of someone else (even a virtual other) may change our perception of the environment, perceiving it in terms of the resources or possibilities for actions held collectively, rather than individually (Kruger, in press). Furthermore, we suggest that this modulatory effect of social gaze on action control in control participants might be related to social cues triggering motor preparatory programs that may help to coordinate one's actions with those of someone else. 
Consistent with this interpretation, a functional magnetic resonance imaging (fMRI) study from our lab using an abridged version of the social SRC paradigm in a group of healthy participants indeed demonstrated that performing incongruent actions in the presence of a virtual other (as compared to performing them in response to a non-social stimulus) leads to a differential increase of neural activity in brain regions known to be involved in action monitoring and response inhibition (anterior cingulate cortex and inferior frontal gyrus), but also areas previously implicated in social cognition (dorso-medial prefrontal cortex; see Schilbach et al., 2010, for details). Taken together, these results highlight that performing actions in an - albeit minimal - social context significantly affects mechanisms of action control. This may play an important role in everyday social interaction, as this could help to automatically adjust to and coordinate one's own actions with those of someone else (Chartrand and Bargh, 1999; Schilbach et al., 2008a).

\section{'Immunity' from the effects of a gaze-based social context on action control in high-functioning autism}

Autism is characterized by impairments of social interaction which, we assume, are closely related to difficulties automatically responding and adjusting to socially relevant nonverbal cues, but also to impairments of executive function and action planning possibly as a result of deficits in inhibitory control and set shifting (Schmitz et al., 2006). In line with other studies, which provide evidence for an 'immunity' of autistic individuals to mechanisms of interpersonal motor alignment evoked by gaze cues (e.g. Becchio et al., 2007) as compared to intact explicit social cognitive abilities (Senju et al., 2009), we hypothesized that individuals with HFA would not be susceptible to the modulatory effect of gaze cues in our social SRC task as observed in the control group. Specifically, we hypothesized that the social stimulus would not lead to a reduction of the incongruency effect in the HFA group.

Consistent with this hypothesis, our investigation did reveal that the RT incongruency costs are not significantly affected by the stimulus type in the HFA group. While the social stimuli led to less pronounced RT costs in the control group, this was not the case in the HFA group. Furthermore, it is important to note the absence of a main effect of group, which suggests that more general impairments of action control assumed to be of relevance in HFA did not have an effect on task performance in this study. Also, our paradigm ensured that patients had to fixate on the eye region of the face stimulus in order to be able to respond. Also, no differences between groups were observed with respect to correctness scores, which further highlights that the groups were well matched with respect to overall cognitive abilities.

Our findings are, therefore, consistent with previous work, which has shown that both adolescents with autism and a comparison group are quicker to detect targets that are preceded by an eye movement cue congruent with target location, compared to targets preceded by an incongruent eye movement cue (Swettenham et al., 2003). Furthermore, the findings by Senju et al. (2004) - using a cueing paradigm similar to the one of Swettenham and colleagues - also seem to be consistent with our results in that the authors describe typically developed children locating targets cued by eye gaze more quickly than those cued by an arrow. Senju et al. also report that in the autistic group both social and non-social cues shifted attention to the cued location, which the authors interpret as a failure of showing preferential sensitivity to the social cue. Crucially, our results go beyond those findings by demonstrating that individuals with HFA do exhibit RT facilitation for both social and non-social stimuli, but do not benefit from the presence of a virtual other when they are asked to generate a spatially incongruent response as indexed by comparable incongruency effects for both social and non-social stimuli.

Drawing upon our above described interpretation of adjustments to the virtual character's perspective in pragmatic terms leading to less pronounced incongruency costs for the face stimulus, it 
could be argued that individuals with autism may not pick up the social affordances (i.e. the possibilities for action provided by others) that allow for a less pronounced reaction time increase in the comparison group. On the neural level this difference might be reflected by a dysconnectivity of brain regions relevant for the perception of gaze shifts (higher-order visual cortices and the temporo-parietal junction; e.g. Pelphrey and Carter, 2008; Sani et al., 2010) and brain regions involved in motor preparation and execution (e.g. Nummenmaa et al., 2010; Caspers et al., 2010) in HFA. Future research using our social SRC task for functional neuroimaging purposes and analyses of functional and effective connectivity may help to directly address this issue.

Taken together, we suggest that our findings are consistent with the proposal of HFA being primarily characterized by difficulties in the automatic integration of social information during action generation (i.e. disturbances of 'online' social cognition), while more explicit measures of 'offline' social cognition may be intact in HFA, possibly as a result of compensatory strategies (Schilbach, 2010; cf. Senju et al., 2009). Whereas control participants appear to respond to the virtual agent's gaze by automatically adjusting to its perspective, leading to a performance benefit as compared to the object stimulus, our results suggest no such difference in HFA.

\section{Conclusions}

Our findings demonstrate that a gaze-mediated social context significantly influences action control in healthy participants, but does not do so in individuals with HFA. While healthy participants appear to benefit from the presence of a virtual other while performing an SRC task leading to less pronounced incongruency effects, individuals with HFA appear to be unfazed. We suggest that the observed modulatory effects of gaze cues on action control might be relevant for processes of interpersonal coordination in everyday life and could rely on mechanisms of automatic perspectivetaking. Such mechanisms of motor-based reactivity could promote interpersonal coordination by helping to constitute a pragmatic common ground during interaction with others. In contrast to this, individuals with autism do not benefit from social cues when generating actions, which might be taken to suggest that patients do not spontaneously adjust to the perspective of someone else, which precludes any behavioral benefit (or disadvantage) this form of interpersonal alignment may impart.

Future research should continue to bring into focus how implicit mechanisms of interpersonal alignment could be seen as fundamental for social functioning as both clinical intuition and recent research findings suggest that impairments of social cognition in HFA may result from patients' inability to intuitively respond to and automatically integrate socially relevant nonverbal cues when directly engaged in 'online' social interaction as compared to situations in which they merely observe others 'offline'. Consequently, it could be these implicit mechanisms of interpersonal alignment - possibly at the very heart of direct everyday social encounters - which turn out to be the elements of social cognition that individuals with autism find most difficult.

\section{References}

Baron-Cohen, S. (2003) The Essential Difference: The Truth about the Male and Female Brain. New York: Basic Books.

Bayliss, A.P., Paul, M.A., Cannon, P.R. \& Tipper, S.P. (2006) 'Gaze Cuing and Affective Judgments of Objects: I Like What You Look at’, Psychon Bull Rev 13(6): 1061-1066.

Becchio, C., Bertone, C. \& Castiello, U. (2008) 'How the Gaze of Others Influences Object Processing', Trends Cogn Sci 12(7): 254-258.

Becchio, C., Pierno, A., Mari, M., Lusher, D. \& Castiello, U. (2007) 'Motor Contagion from Gaze: The Case of Autism', Brain 130(Pt 9): 2401-2411. 
Beck, A.T. \& Steer, R.A. (1987) Beck Depression Inventory - Manual. San Antonio, TX: The Psychological Corporation.

Caspers, S., Zilles, K., Laird, A.R. \& Eickhoff, S.B. (2010) 'ALE Meta-Analysis of Action Observation and Imitation in the Human Brain', Neuroimage 50(3): 1148-67.

Cattaneo, L., Fabbri-Destro, M., Boria, S., Pieraccini, C., Monti, A., et al. (2007) 'Impairment of Actions Chains in Autism and its Possible Role in Intention Understanding', Proc Natl Acad Sci U S A 104(45): 17825-17830.

Chartrand, T.L. \& Bargh, J.A. (1999) 'The Chameleon Effect: The Perception-Behavior Link and Social Interaction', J Pers Soc Psychol 76(6): 893-910.

Costall, A. (1995) 'Socializing Affordances', Theory Psychology 5(4): 467-481.

Emery, N.J. (2000) 'The Eyes Have It: The Neuroethology, Function and Evolution of Social Gaze', Neurosci Biobehav Rev 24(6): 581-604.

Farroni, T., Csibra, G., Simion, F. \& Johnson, M.H. (2002) 'Eye Contact Detection in Humans from Birth', Proc Natl Acad Sci U S A 99(14): 9602-9605.

Grossmann, T., Johnson, M.H., Farroni, T. \& Csibra, G. (2007) 'Social Perception in the Infant Brain: Gamma Oscillatory Activity in Response to Eye Gaze', Soc Cogn Affect Neurosci 2(4): 284-291.

Hoehl, S., Reid, V.M., Parise, E., Handl, A., Palumbo, L. \& Striano, T. (2009) 'Looking at Eye Gaze Processing and Its Neural Correlates in Infancy-Implications for Social Development and Autism Spectrum Disorder', Child Dev 80(4): 968-985.

Iacoboni, M., Woods, R.P. \& Mazziotta, J.C. (1996) 'Brain-Behavior Relationships: Evidence from Practice Effects in Spatial Stimulus-Response Compatibility', J Neurophysiol 76(1): 321-331.

Klin, A., Jones, W., Schultz, R. \& Volkmar, F. (2003) 'The Enactive Mind, or from Actions to Cognition: Lessons from Autism', Philos Trans R Soc Lond B Biol Sci 358(1430): 345-360.

Kruger, J. (in press) 'Extended Cognition and the Space of Social Interaction', Consc Cog.

Kuzmanovic, B., Georgescu, A.L., Eickhoff, S.B., Shah, N. J., Bente, G., et al. (2009) 'Duration Matters: Dissociating Neural Correlates of Detection and Evaluation of Social Gaze', Neuroimage 46(4): 1154-1163.

Lehrl, S., Triebig, G. \& Fischer, B. (1995) 'Multiple Choice Vocabulary Test MWT as a Valid and Short Test to Estimate Premorbid Intelligence', Acta Neurol Scand 91(5): 335-345.

Macrae, C.N., Hood, B.M., Milne, A.B., Rowe, A.C. \& Mason, M.F. (2002) 'Are You Looking at Me? Eye Gaze and Person Perception', Psychol Sci 13(5): 460-464.

Matsumoto, E., Misaki, M. \& Miyauchi, S. (2004) 'Neural Mechanisms of Spatial Stimulus-Response Compatibility: The Effect of Crossed-Hand Position', Exp Brain Res 158(1): 9-17.

Nummenmaa, L., Hyona, J. \& Hietanen, J.K. (2009) 'I'll Walk This Way: Eyes Reveal the Direction of Locomotion and Make Passersby Look and Go the Other Way', Psychol Sci.

Pelphrey, K.A. \& Carter, E.J. (2008) 'Brain Mechanisms for Social Perception: Lessons from Autism and Typical Development', Ann N Y Acad Sci 1145: 283-299.

Proctor, R.W. \& Reeve, T. (1990) Stimulus-Response Compatibility: An Integrated Perspective. Amsterdam: Elsevier.

Quadflieg, S., Mason, M.F. \& Macrae, C.N. (2004) 'The Owl and the Pussycat: Gaze Cues and Visuospatial Orienting', Psychon Bull Rev 11(5): 826-831.

Rinehart, N.J., Bellgrove, M.A., Tonge, B.J., Brereton, A.V., Howells-Rankin, D. \& Bradshaw, J.L. (2006) 'An Examination of Movement Kinematics in Young People with High-Functioning Autism and Asperger's Disorder: Further Evidence for a Motor Planning Deficit', J Autism Dev Disord 36(6): 757-767.

Ristic, J. \& Kingstone, A. (2005) 'Taking Control of Reflexive Social Attention', Cognition 94(3): B55-65.

Sani, L., Ricciardi, E., Gentili, C., Vanello, N., Haxby, J.V. \& Pietrini, P. (2010), Effects of Visual Experience on the Human MT+ Functional Connectivity Networks: An fMRI Study of Motion Perception in Sighted and Congenitally Blind Individuals', Front Syst Neurosci 4: 159.

Schilbach, L., Eickhoff, S.B., Cieslik, E., Shah, N.J., Fink, G.R. \& Vogeley, K. (2010) 'Eyes on Me. An fMRI Study of the Effects of Social Gaze on Action Control', Soc Cog Aff Neurosci [Epub ahead of print].

Schilbach, L. (2010) ,A Second-Person Approach to Other Minds', Nat Rev Neurosci 11(6): 449. 
Schilbach, L., Eickhoff, S.B., Mojzisch, A. \& Vogeley, K. (2008a) 'What's in a Smile? Neural Correlates of Facial Embodiment during Social Interaction', Soc Neurosci 3(1): 37-50.

Schilbach, L., Eickhoff, S.B., Rotarska-Jagiela, A., Fink, G.R. \& Vogeley, K. (2008b) 'Minds at Rest? Social Cognition as the Default Mode of Cognizing and its Putative Relationship to the "Default System" of the Brain', Conscious Cogn 17(2): 457-467.

Schmidt, K.H. \& Metzler, P. (1992) Wortschatztest (WST). Weinheim: Beltz Test GmbH.

Schmitz, N., Rubia, K., Daly, E., Smith, A., Williams, S. \& Murphy, D.G. (2006) 'Neural Correlates of Executive Function in Autistic Spectrum Disorders', Biol Psychiatry 59(1): 7-16.

Senju, A. \& Johnson, M.H. (2009) 'The Eye Contact Effect: Mechanisms and Development', Trends Cogn Sci 13(3): 127-134.

Senju, A., Tojo, Y., Dairoku, H. \& Hasegawa, T. (2004) 'Reflexive Orienting in Response to Eye Gaze and an Arrow in Children with and without Autism', $J$ Child Psychol Psychiatry 45(3): 445-458.

Senju, A., Southgate, V., White, S. \& Frith, U. (2009) 'Mindblind Eyes: An Absence of Spontaneous Theory of Mind in Asperger Syndrome', Science 325(5942): 883-885.

Shepherd, S.V. (in press) 'Following Gaze: Gaze-Following Behavior as a Window into Social Cognition', Frontiers in Integrative Neuroscience.

Stewart, M.E., Barnard, L., Pearson, J., Hasan, R. \& O’Brien, G. (2006) 'Presentation of Depression in Autism and Asperger Syndrome: A Review', Autism 10(1): 103-116.

Suslow, T. (2009) 'Estimating Verbal Intelligence in Unipolar Depression: Comparison of Word Definition and Word Recognition', Nord J Psychiatry 63(2): 120-123.

Swettenham, J., Condie, S., Campbell, R., Milne, E. \& Coleman, M. (2003) 'Does the Perception of Moving Eyes Trigger Reflexive Visual Orienting in Autism?' Philos Trans R Soc Lond B Biol Sci 358(1430): 325-334.

Tversky, B. \& Hard, B.M. (2009) 'Embodied and Disembodied Cognition: Spatial Perspective-Taking', Cognition 110(1): 124-129.

von Grunau, M. \& Anston, C. (1995) 'The Detection of Gaze Direction: A Stare-in-the-Crowd Effect', Perception 24(11): 1297-1313.

Wheelwright, S., Baron-Cohen, S., Goldenfeld, N., Delaney, J., Fine, D., et al. (2006) 'Predicting Autism Spectrum Quotient (AQ) from the Systemizing Quotient-Revised (SQ-R) and Empathy Quotient (EQ)', Brain Res 1079(1): 47-56.

Zajonc, R.B. (1965) 'Social Facilitation', Science 149: 269-274.

Zwickel, J. (2009) 'Agency Attribution and Visuospatial Perspective Taking', Psychon Bull Rev 16(6): 10891093. DOI: 10.3758/PBR.16.6.1089 